American J. of Engineering and Applied Sciences 2 (4): 683-693, 2009

ISSN 1941-7020

(C) 2009 Science Publications

\title{
Converting of Matter to Nuclear Energy by AB-Generator
}

\author{
Alexander Bolonkin \\ C and R, 1310 Avenue R, No. F-6, Brooklyn, NY 11229, USA
}

\begin{abstract}
Problem statement: Researcher offered a new nuclear generator which allowed to convert any matter to nuclear energy in accordance with Einstein equation $\mathrm{E}=\mathrm{mc}^{2}$. The method was based upon tapping the energy potential of a Micro Black Hole (MBH) and Hawking radiation created by this MBH. Researcher did not meet the idea and its research in literature to develop the method for getting a cheap energy. Approach: As is well-known, vacuum continuously produced virtual pairs of particles and antiparticles, in particular, photons and anti-photons. MBH event horizon allowed separating them. Anti-photons can be moved to $\mathrm{MBH}$ and be annihilated, decreasing mass of $\mathrm{MBH}$, resulting photons leave the $\mathrm{MBH}$ neighborhood as Hawking radiation. The offered nuclear generator (named by Researcher as AB-generator) utilized Hawking radiation and injected the matter into $\mathrm{MBH}$ and kept $\mathrm{MBH}$ in a stable state with near-constant mass. Results: AB-generator can be produced gigantic energy outputs and should be cheaper than a conventional electric station by a factor of hundreds of times. One also may be used in aerospace as a photon rocket or as a power source for many vehicles. Conclusion: Many scientists expect Large Hadron Collider at CERN may be produced one MBH every second. A technology to capture them may be developed; than they may be used for the AB-generator.
\end{abstract}

Key words: Production of nuclear energy, micro black hole, energy AB-generator, photon rocket

\section{INTRODUCTION}

Black hole: In general relativity, a black hole (Fig. 1-4) is a region of space in which the gravitational field is so powerful that nothing, including light, can escape its pull. The black hole has a one-way surface, called the event horizon, into which objects can fall, but out of which nothing can come out. It is called "black" because it absorbs all the light that hits it, reflecting nothing, just like a perfect blackbody in thermodynamics.

Despite its invisible interior, a black hole can reveal its presence through interaction with other matter.

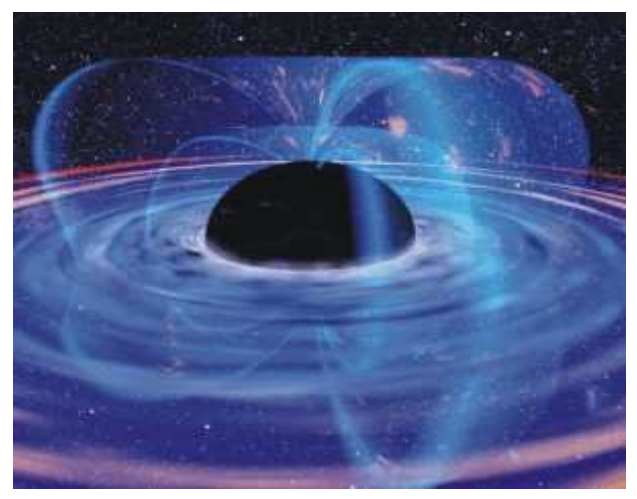

Fig. 1: Artist's conception of a stellar mass black hole. Credit NASA
A black hole can be inferred by tracking the movement of a group of stars that orbit a region in space which looks empty. Alternatively, one can see gas falling into a relatively small black hole, from a companion star. This gas spirals inward, heating up to very high temperature and emitting large amounts of radiation that can be detected from earthbound and earth-orbiting telescopes. Such observations have resulted in the general scientific consensus that, barring a breakdown in our understanding of nature, black holes do exist in our universe.

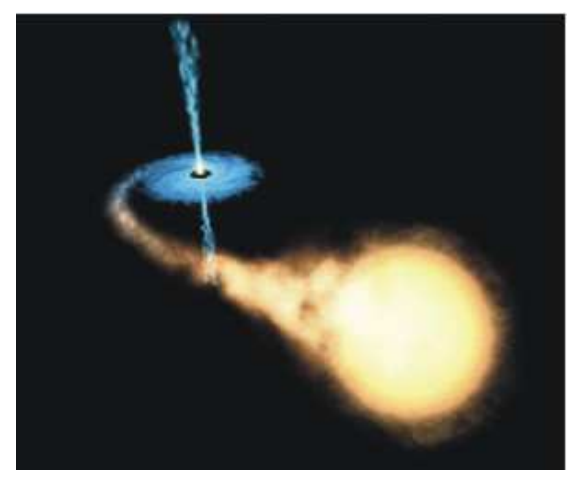

Fig. 2: Artist's impression of a binary system consisting of a black hole and a main sequence star. The black hole is drawing matter from the main sequence star via an accretion disk around it and some of this matter forms a gas jet 


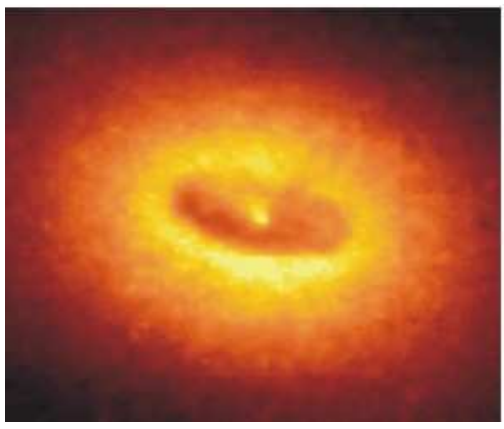

Fig. 3: Ring around a suspected black hole in galaxy NGC 4261. Date: Nov.1992. Courtesy of space telescope science

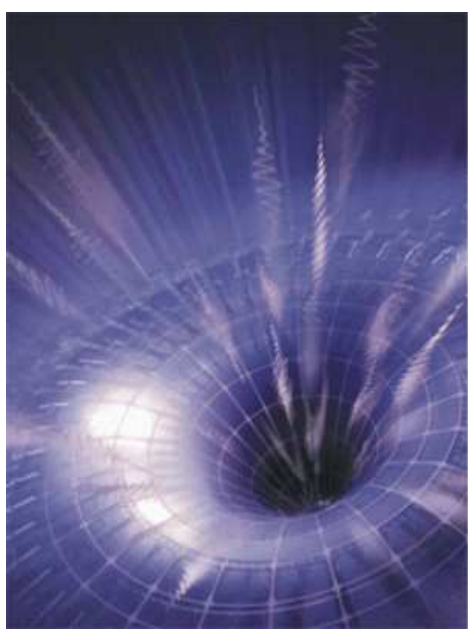

Fig. 4: Artist's rendering showing the space-time contours around a black hole. Credit NASA

It is impossible to directly observe a black hole. However, it is possible to infer its presence by its gravitational action on the surrounding environment, particularly with micro-quasars and active galactic nuclei, where material falling into a nearby black hole is significantly heated and emits a large amount of X-ray radiation. This observation method allows astronomers to detect their existence. The only objects that agree with these observations and are consistent within the framework of general relativity are black holes.

A black hole has only three independent physical properties: mass, charge and angular momentum.

In astronomy black holes are classed as:

- Supermassive-contain hundreds of thousands to billions of solar masses and are thought to exist in the center of most galaxies, including the Milky Way
- Intermediate-contain thousands of solar masses

- Micro (also mini black holes)-have masses much less than that of a star. At these sizes, quantum mechanics is expected to take effect. There is no known mechanism for them to form via normal processes of stellar evolution, but certain inflationary scenarios predict their production during the early stages of the evolution of the universe

According to some theories of quantum gravity they may also be produced in the highly energetic reaction produced by cosmic rays hitting the atmosphere or even in particle accelerators such as the Large Hadron Collider. The theory of Hawking radiation predicts that such black holes will evaporate in bright flashes of gamma radiation. NASA's Fermi Gamma-ray Space Telescope satellite (formerly GLAST) launched in 2008 is searching for such flashes.

The defining feature of a black hole is the appearance of an event horizon; a boundary in spacetime beyond which events cannot affect an outside observer.

Since the event horizon is not a material surface but rather merely a mathematically defined demarcation boundary, nothing prevents matter or radiation from entering a black hole, only from exiting one.

For a non rotating (static) black hole, the Schwarzschild radius delimits a spherical event horizon. The Schwarzschild radius of an object is proportional to the mass. Rotating black holes have distorted, non-spherical event horizons. The description of black holes given by general relativity is known to be an approximation and it is expected that quantum gravity effects become significant near the vicinity of the event horizon. This allows observations of matter in the vicinity of a black hole's event horizon to be used to indirectly study general relativity and proposed extensions to it.

Though black holes themselves may not radiate energy, electromagnetic radiation and matter particles may be radiated from just outside the event horizon via Hawking radiation.

At the center of a black hole lies the singularity, where matter is crushed to infinite density, the pull of gravity is infinitely strong and space-time has infinite curvature. This means that a black hole's mass becomes entirely compressed into a region with zero volume. This zero-volume, infinitely dense region at the center of a black hole is called a gravitational singularity.

The singularity of a non-rotating black hole has zero length, width and height; a rotating black hole's is smeared out to form a ring shape lying in the plane of 
rotation. The ring still has no thickness and hence no volume.

The photon sphere is a spherical boundary of zero thickness such that photons moving along tangents to the sphere will be trapped in a circular orbit. For nonrotating black holes, the photon sphere has a radius 1.5 times the Schwarzschild radius. The orbits are dynamically unstable, hence any small perturbation (such as a particle of in-falling matter) will grow over time, either setting it on an outward trajectory escaping the black hole or on an inward spiral eventually crossing the event horizon.

Rotating black holes are surrounded by a region of space-time in which it is impossible to stand still, called the ergo-sphere. Objects and radiation (including light) can stay in orbit within the ergo-sphere without falling to the center.

Once a black hole has formed, it can continue to grow by absorbing additional matter. Any black hole will continually absorb interstellar dust from its direct surroundings and omnipresent cosmic background radiation. Much larger contributions can be obtained when a black hole merges with other stars or compact objects.

Hawking radiation: In 1974, Stephen Hawking showed that black holes are not entirely black but emit small amounts of thermal radiation ${ }^{[1]}$. He got this result by applying quantum field theory in a static black hole background. The result of his calculations is that a black hole should emit particles in a perfect black body spectrum. This effect has become known as Hawking radiation. Since Hawking's result many others have verified the effect through various methods. If his theory of black hole radiation is correct then black holes are expected to emit a thermal spectrum of radiation and thereby lose mass, because according to the theory of relativity mass is just highly condensed energy $\left(E=\mathrm{mc}^{2}\right)$. Black holes will shrink and evaporate over time. The temperature of this spectrum (Hawking temperature) is proportional to the surface gravity of the black hole, which in turn is inversely proportional to the mass. Large black holes, therefore, emit less radiation than small black holes.

On the other hand if a black hole is very small, the radiation effects are expected to become very strong. Even a black hole that is heavy compared to a human would evaporate in an instant. A black hole the weight of a car $\left(\sim 10^{-24} \mathrm{~m}\right)$ would only take a nanosecond to evaporate, during which time it would briefly have a luminosity more than 200 times that of the sun. Lighter black holes are expected to evaporate even faster, for example a black hole of mass $1 \mathrm{TeV} / \mathrm{c}^{2}$ would take less than $10^{-88} \mathrm{sec}$ to evaporate completely. Of course, for such a small black hole quantum gravitation effects are expected to play an important role and could evenalthough current developments in quantum gravity do not indicate so-hypothetically make such a small black hole stable.

Micro black holes: Gravitational collapse is not the only process that could create black holes. In principle, black holes could also be created in high energy collisions that create sufficient density. Since classically black holes can take any mass, one would expect micro black holes to be created in any such process no matter how low the energy. However, to date, no such events have ever been detected either directly or indirectly as a deficiency of the mass balance in particle accelerator experiments. This suggests that there must be a lower limit for the mass of black holes.

Theoretically this boundary is expected to lie around the Planck mass $\left(\sim 10^{19} \mathrm{GeV} / \mathrm{c}^{2}, \mathrm{~m}_{\mathrm{p}}=2.176410^{-8} \mathrm{~kg}\right)$, where quantum effects are expected to make the theory of general relativity break down completely. This would put the creation of black holes firmly out of reach of any high energy process occurring on or near the Earth. Certain developments in quantum gravity however suggest that this bound could be much lower. Some braneworld scenarios for example put the Planck mass much lower, maybe even as low as $1 \mathrm{TeV}$. This would make it possible for micro black holes to be created in the high energy collisions occurring when cosmic rays hit the Earth's atmosphere, or possibly in the new Large Hadron Collider at CERN. These theories are however very speculative and the creation of black holes in these processes is deemed unlikely by many specialists.

Smallest possible black hole: To make a black hole one must concentrate mass or energy sufficiently that the escape velocity from the region in which it is concentrated exceeds the speed of light. This condition gives the Schwarzschild radius, $r_{o}=2 G M / c^{2}$, where $G$ is Newton's constant and $\mathrm{c}$ is the speed of light, as the size of a black hole of mass $\mathrm{M}$. On the other hand, the Compton wavelength, $\lambda=\mathrm{h} / \mathrm{Mc}$, where $\mathrm{h}$ is Planck's constant, represents a limit on the minimum size of the region in which a mass $M$ at rest can be localized. For sufficiently small $\mathrm{M}$, the Compton wavelength exceeds the Schwarzschild radius and no black hole description exists. This smallest mass for a black hole is thus approximately the Planck mass, which is about $2 \times 10^{-8} \mathrm{~kg}$ or $1.2 \times 10^{19} \mathrm{GeV} / \mathrm{c}^{2}$. 
Any primordial black holes of sufficiently low mass will Hawking evaporate to near the Planck mass within the lifetime of the universe. In this process, these small black holes radiate away matter. A rough picture of this is that pairs of virtual particles emerge from the vacuum near the event horizon, with one member of a pair being captured and the other escaping the vicinity of the black hole. The net result is the black hole loses mass (due to conservation of energy). According to the formulae of black hole thermodynamics, the more the black hole loses mass the hotter it becomes and the faster it evaporates, until it approaches the Planck mass. At this stage a black hole would have a Hawking temperature of $\mathrm{T}_{\mathrm{P}} / 8 \pi\left(5.6 \times 10^{32} \mathrm{~K}\right)$, which means an emitted Hawking particle would have an energy comparable to the mass of the black hole. Thus a thermodynamic description breaks down. Such a miniblack hole would also have an entropy of only $4 \pi$ nats, approximately the minimum possible value.

At this point then, the object can no longer be described as a classical black hole and Hawking's calculations also break down. Conjectures for the final fate of the black hole include total evaporation and production of a Planck mass-sized black hole remnant. If intuitions about quantum black holes are correct, then close to the Planck mass the number of possible quantum states of the black hole is expected to become so few and so quantized that its interactions are likely to be quenched out. It is possible that such Planck-mass black holes, no longer able either to absorb energy gravitationally like a classical black hole because of the quantized gaps between their allowed energy levels, nor to emit Hawking particles for the same reason, may in effect be stable objects. They would in effect be WIMPs, weakly interacting massive particles; this could explain dark matter.

Creation of micro black holes: Production of a black hole requires concentration of mass or energy within the corresponding Schwarzschild radius. In familiar three-dimensional gravity, the minimum such energy is $10^{19} \mathrm{GeV}$, which would have to be condensed into a region of approximate size $10^{-33} \mathrm{~cm}$. This is far beyond the limits of any current technology; the Large Hadron Collider (LHC) has a design energy of $14 \mathrm{TeV}$. This is also beyond the range of known collisions of cosmic rays with Earth's atmosphere, which reach center of mass energies in the range of hundreds of $\mathrm{TeV}$. It is estimated that to collide two particles to within a distance of a Planck length with currently achievable magnetic field strengths would require a ring accelerator about 1000 light years in diameter to keep the particles on track.
Some extensions of present physics posit the existence of extra dimensions of space. In higherdimensional spacetime, the strength of gravity increases more rapidly with decreasing distance than in three dimensions. With certain special configurations of the extra dimensions, this effect can lower the Planck scale to the TeV range. Examples of such extensions include large extra dimensions, special cases of the RandallSundrum model and String theory configurations. In such scenarios, black hole production could possibly be an important and observable effect at the LHC.

Virtual particles: In physics, a virtual particle is a particle that exists for a limited time and space, introducing uncertainty in their energy and momentum due to the Heisenberg Uncertainty Principle. Vacuum energy can also be thought of in terms of virtual particles (also known as vacuum fluctuations) which are created and destroyed out of the vacuum. These particles are always created out of the vacuum in particle-antiparticle pairs, which shortly annihilate each other and disappear. However, these particles and antiparticles may interact with others before disappearing. The net energy of the Universe remains zero so long as the particle pairs annihilate each other within Planck time.

Virtual particles are also excitations of the underlying fields, but are detectable only as forces.

The creation of these virtual particles near the event horizon of a black hole has been hypothesized by physicist Stephen Hawking to be a mechanism for the eventual "evaporation" of black holes. Since these particles do not have a permanent existence, they are called virtual particles or vacuum fluctuations of vacuum energy.

An important example of the "presence" of virtual particles in a vacuum is the Casimir effect. Here, the explanation of the effect requires that the total energy of all of the virtual particles in a vacuum can be added together. Thus, although the virtual particles themselves are not directly observable in the laboratory, they do leave an observable effect: their zero-point energy results in forces acting on suitably arranged metal plates or dielectrics. Thus, virtual particles are often popularly described as coming in pairs, a particle and antiparticle, which can be of any kind.

The evaporation of a black hole is a process dominated by photons, which are their own antiparticles and are uncharged.

The uncertainty principle in the form $\Delta \mathrm{E} \Delta \mathrm{t} \geq \hbar$ implies that in the vacuum one or more particles with energy $\Delta \mathrm{E}$ above the vacuum may be created for a short time $\Delta \mathrm{t}$. These virtual particles are included in the definition of the vacuum. 
Am. J. Engg. \& Applied Sci., 2 (4): 683-693, 2009

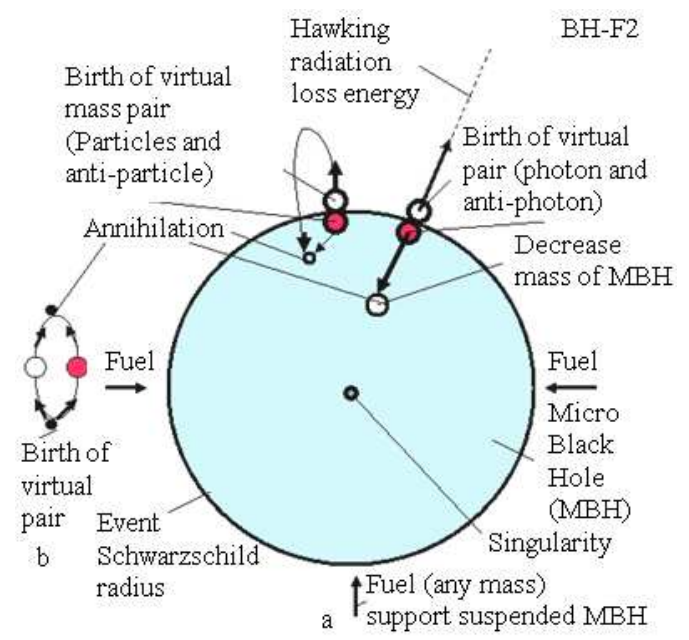

Fig. 5: Hawking radiation. (a): Virtual particles at even horizon. (b): Virtual particles out even horizon (in conventional space)

Vacuum energy is an underlying background energy that exists in space even when devoid of matter (known as free space). The vacuum energy is deduced from the concept of virtual particles, which are themselves derived from the energy-time uncertainty principle. Its effects can be observed in various phenomena (such as spontaneous emission, the Casimir effect, the van der Waals bonds, or the Lamb shift) and it is thought to have consequences for the behavior of the Universe on cosmological scales.

\section{AB-generator of nuclear energy and some innovations:}

Simplified explanation of MBH radiation and work of AB-generator (Fig. 5): As known, the vacuum continuously produces, virtual pairs of particles and antiparticles, in particular, photons and anti-photons. In conventional space they exist only for a very short time, then annihilate and return back to nothingness. The MBH event horizon, having very strong super-gravity, allows separation of the particles and anti particles, in particular, photons and anti-photons. Part of the antiphotons move into the $\mathrm{MBH}$ and annihilate with photons decreasing the mass of the MBH and return back a borrow energy to vacuum. The free photons leave from the MBH neighborhood as Hawking radiation. That way the $\mathrm{MBH}$ converts any conventional matter to Hawking radiation which may be converted to heat or electric energy by the ABGenerator. This AB-Generator utilizes the produced Hawking radiation and injects the matter into the MBH while maintaining the MBH in stable suspended state.

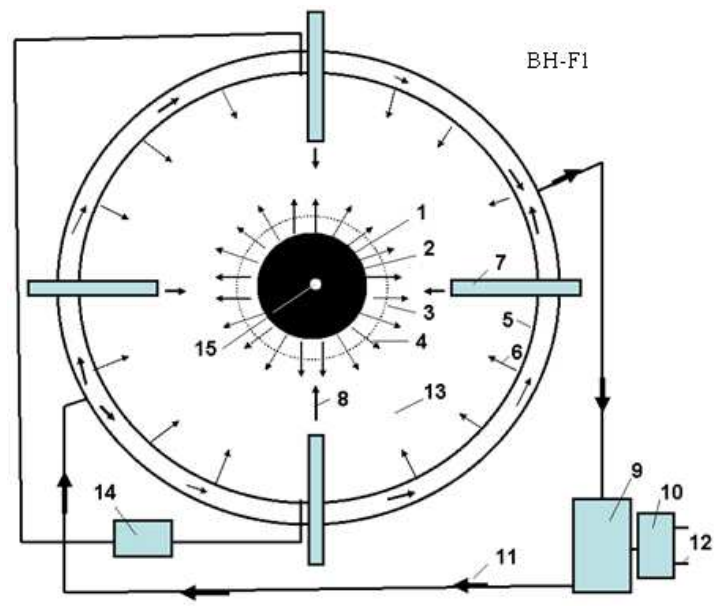

Fig. 6: Offered nuclear-vacuum energy ABGenerator. Notations: (1): Micro Black Hole (MBH), (2): Event horizon (Schwarzschild radius), (3): Photon sphere, (4): Black hole radiation, (5): Radiation reflector, antenna and heater (cover sphere), (6): Back (reflected) radiation from radiation reflector 5, (7): Fuel (plasma, protons, electrons, ions, matter) gun (focusing accelerator), (8): Matter injected to MBH (fuel for Micro Black hole), (9): Heat engine (for example, gas, vapor turbine), (10): Electric generator connected to heat engine 9, (11): Coolant (heat transfer agent to the heat machine 9), (12): Electric line, (13): Internal vacuum, (14): Customer of electricity from antenna 5, (15): Singularity

Note: The photon does NOT have rest mass. Therefore a photon can leave the MBH's neighborhood (if it is located beyond the event horizon). All other particles having a rest mass and speed less than light speed cannot leave the Black Hole. They cannot achieve light speed because their mass at light speed equals infinity and requests infinite energy for it's escape-an impossibility.

Description of AB-generator: The offered nuclear energy AB-Generator is shown in Fig. 6. That includes the Micro Black Hole (MBH) 1 suspended within a spherical radiation reflector and heater 5. The MBH is supported (and controlled) at the center of sphere by a fuel (plasma, proton, electron, matter) gun 7. This ABGenerator also contains the 9-heat engine (for example, gas, vapor turbine), 10-electric generator, 11-coolant (heat transfer agent), an outer electric line 12, internal electric generator (5 as antenna) with customer 14. 
Work: The generator works the following way. MBH, by selective directional input of matter, is levitated in captivity and produces radiation energy 4. That radiation heats the spherical reflector-heater 5 . The coolant (heat transfer agent) 11 delivers the heat to a heat machine 9 (for example, gas, vapor turbine). The heat machine rotates an electric generator 10 that produces the electricity to the outer electric line 12 . Part of MBH radiation may accept by sphere 5 (as antenna) in form of electricity. The control fuel guns inject the matter into $\mathrm{MBH}$ and do not allow bursting of the $\mathrm{MBH}$. This action also supports the MBH in isolation, suspended from dangerous contact with conventional matter. They also control the MBH size and the energy output. Any matter may be used as the fuel, for example, accelerated plasma, ions, protons, electrons, micro particles, etc. The MBH may be charged and rotated. In this case the MBH may has an additional suspension by control charges located at the ends of fuel guns or (in case of the rotating charged $\mathrm{MBH}$ ) may have an additional suspension by the control electric magnets located on the ends of fuel guns or at points along the reflector-heater sphere.

Innovations, features, advantages and same research results: Some problems and solutions offered by the author include the following:

- A practical (the MBH being obtained and levitated, details of which are beyond the scope of this study) method and installation for converting any conventional matter to energy in accordance with Einstein's equation $\mathrm{E}=\mathrm{mc}^{2}$

- MBHs may produce gigantic energy and this energy is in the form of dangerous gamma radiation. The author shows how this dangerous gamma radiation Doppler shifts when it moves against the MBH gravity and converts to safely tapped short radio waves

- The MBH of marginal mass has a tendency to explode (through quantum evaporation, very quickly radiating its mass in energy). The $\mathrm{AB}$ Generator automatically injects metered amounts of matter into the MBH and keeps the MGH in a stable state or grows the MBH to a needed size, or decreases that size, or temporarily turns off the $\mathrm{AB}$-generator (decreases the $\mathrm{MBH}$ to a Planck black hole)

- Author shows the radiation flux exposure of ABgenerator (as result of $\mathrm{MBH}$ exposure) is not dangerous because the generator cover sphere has a vacuum and the $\mathrm{MBH}$ gravity gradient decreases the radiation energy
- The MBH may be supported in a levitated (noncontact) state by generator fuel injectors

Theory of AB-generator: Below there are main equations for computation the conventional Black Hole $(\mathrm{BH})$ and $\mathrm{AB}$-generator.

\section{General theory of black hole:}

- Power produced by BH is:

$\mathrm{P}=\frac{\hbar \mathrm{c}^{6}}{15360 \pi \mathrm{G}^{2}} \frac{1}{\mathrm{M}} \approx 3.56 \cdot 10^{32} \frac{1}{\mathrm{M}}, \mathrm{W}$

where, $\quad \hbar=\mathrm{h} / 2 \pi=1.0546 \cdot 10^{-34} \mathrm{~J} \mathrm{~s}^{-1} \quad$ is reduced Planck constant, $\mathrm{c}=3 \cdot 10^{8} \mathrm{~m} \mathrm{sec}^{-1}$-light speed, $\mathrm{G}=6.6743 \cdot 10^{-11} \mathrm{~m}^{3} / \mathrm{kg} \cdot \mathrm{s}^{2}$ is gravitation constant, M-mass of $\mathrm{BH}, \mathrm{kg}$

- Temperature of black body corresponding to this radiation is:

$\mathrm{T}=\frac{\hbar \mathrm{c}^{3}}{8 \pi \mathrm{Gk}_{\mathrm{b}}} \frac{1}{\mathrm{M}} \approx 1.23 \cdot 10^{23} \frac{1}{\mathrm{M}}, \mathrm{K}$

where, $\mathrm{k}_{\mathrm{b}}=1.38 .10^{-23} \mathrm{~J} / \mathrm{k}$ is Boltzmann constant

- Energy $E_{p}[J]$ and frequency $v_{o}$ of photon at event horizon are:

$\mathrm{E}_{\mathrm{p}}=\frac{\mathrm{hc^{3 }}}{16 \pi \mathrm{G}} \frac{1}{\mathrm{M}}$,

$v_{0}=\frac{E_{p}}{h}=\frac{c^{3}}{16 \pi G} \frac{1}{M}=8.037 \cdot 10^{33} \frac{1}{M}$,

$\lambda_{0}=\frac{\mathrm{c}}{\mathrm{v}_{0}}=3.73 \cdot 10^{-26} \mathrm{M}$

Where:

c $=310^{8} \mathrm{~m} \mathrm{~s}^{-1}$ is light speed

$\lambda_{\mathrm{o}}=$ Wavelength of photon at even radius, $\mathrm{m}$

$\mathrm{h}=$ Planck constant

- Radius of BH event horizon (Schwarzschild radius) is:

$\mathrm{r}_{0}=\frac{2 \mathrm{G}}{\mathrm{c}^{2}} \mathrm{M}=1.48 \cdot 10^{-27} \mathrm{M}, \mathrm{m}$

- Relative density (ratio of mass $\mathrm{M}$ to volume $\mathrm{V}$ of $\mathrm{BH})$ is:

$\rho=\frac{\mathrm{M}}{\mathrm{V}}=\frac{3 \mathrm{c}^{2}}{32 \pi \mathrm{G}^{3}} \frac{1}{\mathrm{M}^{2}} \approx 7.33 \cdot 10^{79} \frac{1}{\mathrm{M}^{2}}, \mathrm{~kg} \mathrm{~m}^{-3}$ 
Am. J. Engg. \& Applied Sci., 2 (4): 683-693, 2009

- Maximal charge of $\mathrm{BH}$ is:

$\mathrm{Q}_{\text {max }}=5 \cdot 10^{9} \mathrm{eM} \approx 8 \cdot 10^{-10} \mathrm{M}, \quad \mathrm{C}$

where, $\mathrm{e}=-1.610^{-19}$ is charge of electron, $\mathrm{C}$

- Life time of BH is:

$$
\tau=\frac{5120 \pi \mathrm{G}^{2}}{\hbar \mathrm{c}^{4}} \mathrm{M}^{3}=2.527 \cdot 10^{-8} \mathrm{M}^{3}, \mathrm{~s}
$$

- Gravitation around BH ( $\mathrm{r}$ is distance from center) and on event horizon:

$$
\mathrm{g}=\frac{\mathrm{GM}}{\mathrm{r}^{2}}, \quad \mathrm{~g}_{0}=\frac{\mathrm{c}^{4}}{4 \mathrm{G}} \frac{1}{\mathrm{M}}=3 \cdot 10^{42} \frac{1}{\mathrm{M}}, \mathrm{m} \mathrm{s}^{-2}
$$

Developed theory of AB-generator: Below are research and the theory developed by author for estimation and computation of facets of the $\mathrm{AB}$ Generator.

Loss of energy of Hawking photon in BH gravitational field: It is known the theory of a redshift allows estimating the frequency of photon in central gravitational field when it moves TO the gravity center. In this case the photon increases its frequency because photon is accelerated the gravitational field (wavelength decreases). But in our case the photon moves FROM the gravitational center, the gravitational field brakes it and the photon loses its energy. That means its frequency decreases and the wavelength increases. Our photon gets double energy because the black hole annihilates two photons (photon and anti-photon). That way the equation for photon frequency at distance $r>r_{o}$ from center we can write in form:

$$
\frac{v}{v_{0}} \approx 1+\frac{2 \Delta \varphi}{c^{2}}
$$

where, $\Delta \varphi=\varphi-\varphi_{o}$ is difference of the gravity potential. The gravity potential is:

$$
\Delta \varphi=\varphi-\varphi_{0}, \quad \varphi=\frac{\mathrm{GM}}{\mathrm{r}}, \quad \varphi_{0}=\frac{\mathrm{GM}}{\mathrm{r}_{0}}, \quad \mathrm{r}_{0}=\frac{2 \mathrm{GM}}{\mathrm{c}^{2}}
$$

Let us substitute (10) in (9), we get:

$$
\frac{v}{v_{0}} \approx 1+\frac{r_{0}}{r}-\frac{r_{0}}{r_{0}}, \quad \text { or } \quad \frac{v}{v_{0}}=\frac{\lambda_{0}}{\lambda} \approx \frac{r_{0}}{r}
$$

It is known, the energy and mass of photon is:

$$
E_{f}=\hbar \gamma, \quad E_{f}=m_{f} c^{2}, \quad m_{f}=E_{f} / c^{2}
$$

The energy of photon linear depends from its frequency. Reminder: The photon does not have a rest mass.

The relative loss of the photon radiation energy $\xi$ at distance $r$ from $\mathrm{BH}$ and the power $\mathrm{P}_{\mathrm{r}}$ of Hawking radiation at radius $r$ from the $\mathrm{BH}$ center is:

$\xi=\frac{\mathrm{r}_{0}}{\mathrm{r}}, \quad v=\xi v_{0}, \quad \mathrm{P}_{\mathrm{r}}=\xi \mathrm{P}$

The $r_{0}$ is very small and $\xi$ is also very small and $v$ $<<v_{0}$.

The result of an energy loss by Hawking photon in the $\mathrm{BH}$ gravitational field is very important for $\mathrm{AB}$ Generator. The energy of Hawking radiation is very big; we very need to decrease it in many orders. The initial Hawking photon is gamma radiation that is dangerous for people and matter. In $r$ distance the gamma radiation may be converted in the conventional light or radio radiation, which are not dangerous and may be reflected, focused or a straightforward way converted into electricity by antenna.

Reflection hawking radiation back to MBH: For further decreasing the MBH produced energy the part of this energy may be reflected to back in MBH. A conventional mirror may reflect up $0.9 \div 0.99$ of radiation $\left(\xi_{\mathrm{r}}=0.01 \div 0.1, \xi_{\mathrm{r}}\right.$ is a loss of energy in reflecting), the multi layers mirror can reflect up 0.9999 of the monochromatic light radiation $\left(\xi_{\mathrm{r}}=10^{-3} \div 10^{-5}\right)$ and $\mathrm{AB}$-mirror from cubic corner cells offered by author in ${ }^{[2]}$, allows to reflect non-monochromatic light radiation with efficiency up $\xi_{\mathrm{r}}=10^{-13}$ strong back to source. In the last case, the loss of reflected energy is ${ }^{[2]}$ :

$\xi_{\mathrm{r}}=0.00023 \mathrm{al}, \quad \mathrm{l}=\mathrm{m} \lambda, \quad \mathrm{m} \geq 1$

Where:

1 = Size of cube corner cell, $\mathrm{m}$

$\mathrm{m}=$ Number of radiation waves in one sell

$\lambda=$ Wavelength, $\mathrm{m}$

$\mathrm{a}=$ Characteristic of sell material ${ }^{[2]}$. Minimal value a $=10^{-2}$ for glass and $\mathrm{a}=10^{-4}$ for $\mathrm{KCl}$ crystal

The reflection of radiation to back in $\mathrm{MBH}$ is may be important for $\mathrm{MBH}$ stabilization, $\mathrm{MBH}$ storage and MBH 'switch off'.

Useful energy of AB-generator: The useful energy $P_{u}$ $[\mathrm{J}]$ is taken from $\mathrm{AB}-$ Generator is: 
$\mathrm{P}_{\mathrm{u}}=\xi \xi_{\mathrm{r}} \mathrm{P}$

Fuel consumption is:

$\dot{\mathrm{M}}=\mathrm{P}_{\mathrm{u}} / \mathrm{c}^{2}, \mathrm{~kg}$

The fuel consumption is very small. AB-generator is the single method in the World now known which allows full converting reasonably practical conversion of (any!) matter into energy according the Einstein equation $\mathrm{E}=\mathrm{mc}^{2}$.

Specific pressure on $A B-G e n e r a t o r$ cover sphere $p$ $\left[\mathrm{N} \mathrm{m}^{-2}\right]$ and on the surface of MBH $\mathrm{p}_{\mathrm{o}}$ is:

$\mathrm{p}=\frac{\mathrm{kP}_{\mathrm{r}}}{\mathrm{Sc}}=\frac{\mathrm{kP}_{\mathrm{r}}}{4 \pi \mathrm{r}^{2} \mathrm{c}}=2.65 \cdot 10^{-10} \frac{\mathrm{kP}_{\mathrm{r}}}{\mathrm{r}^{2}}$,

$\mathrm{p}_{0}=\frac{\mathrm{P}}{\mathrm{S}_{0} \mathrm{c}}=\frac{\hbar \mathrm{c}^{8}}{15360 \cdot 16 \pi^{2} \mathrm{G}^{4}} \frac{1}{\mathrm{M}^{4}}=1.44 \cdot 10^{28} \frac{1}{\mathrm{M}^{4}}$

Where:

$\mathrm{k}=1$ if the cover sphere absorbs the radiation and $\mathrm{k}$ $\approx 2$ if the cover sphere high reflects the radiation

$\mathrm{S}=$ The internal area of cover sphere, $\mathrm{m}^{2}$

$\mathrm{S}_{0}=$ Surface of event horizon sphere, $\mathrm{m}^{2}$

$\mathrm{p}_{\mathrm{o}}=$ Specific pressure of Hawking radiation on the event horizon surface

Note, the pressure $\mathrm{p}$ on cover sphere is small (see project), but pressure $\mathrm{p}_{\mathrm{o}}$ on event horizon surface is very high.

Mass particles produced on event surface: On event horizon surface may be also produced the mass particles with speed $\mathrm{V}<\mathrm{c}$. Let us take the best case (for leaving the $\mathrm{BH}$ ) when their speed is radially vertical. They cannot leave the BH because their speed $\mathrm{V}$ is less than light speed $\mathrm{c}$. The maximal radius of lifting $\mathrm{r}_{\mathrm{m}}[\mathrm{m}]$ is:

$$
\begin{aligned}
& \mathrm{dV}=-\mathrm{gdt}, \quad \mathrm{dV}=-\frac{\mathrm{g}}{\mathrm{V}} \mathrm{dr}=-\frac{\mathrm{GM}}{\mathrm{V}} \frac{\mathrm{dr}}{\mathrm{r}^{2}}, \\
& \mathrm{r}_{\mathrm{m}}=\frac{2 \mathrm{GM}}{\mathrm{c}^{2}-\mathrm{V}_{0}^{2}}=\frac{\mathrm{r}_{0}}{1-(\mathrm{V} / \mathrm{c})^{2}}
\end{aligned}
$$

Where:

$$
\begin{aligned}
& \mathrm{g}=\text { Gravitational acceleration of } \mathrm{BH}, \mathrm{m} \mathrm{sec}^{-2} \\
& \mathrm{t}=\text { Time, } \mathrm{sec} \\
& \mathrm{r}_{\mathrm{o}}=\mathrm{BH} \text { radius, } \mathrm{m} \\
& \mathrm{V}_{0}=\text { Particle speed on event surface, } \mathrm{m} \mathrm{sec}^{-2}
\end{aligned}
$$

If the $r_{m}$ is less than radius of the cover sphere, the mass particles return to $\mathrm{BH}$ and do not influence the heat flow from $\mathrm{BH}$ to cover sphere. That is in the majority of cases.

Explosion of MBH: The MBH explosion produces the radiation energy:

$\mathrm{E}_{\mathrm{e}}=\mathrm{Mc}^{2}$

MBH has a small mass. The explosion of MBH having $\mathrm{M}=10^{-5} \mathrm{~kg}$ produces $9 \times 10^{11} \mathrm{~J}$. That is energy of about 10 tons of good conventional explosive $\left(10^{7} \mathrm{~J} \mathrm{~kg}^{-1}\right)$. But there is a vacuum into the cover sphere and this energy is presented in radiation form. But in reality only very small part of explosion energy reaches the cover sphere, because the very strong $\mathrm{MBH}$ gravitation field brakes the photons and any mass particles. Find the energy which reaches the cover sphere via:

$$
\begin{aligned}
& \mathrm{dE}=\xi \mathrm{c}^{2} \mathrm{dM}, \quad \xi=\frac{\mathrm{r}_{0}}{\mathrm{r}}, \quad \mathrm{r}_{0}=\frac{2 \mathrm{G}}{\mathrm{c}^{2}} \mathrm{M}, \quad \mathrm{dE}=\frac{2 \mathrm{G}}{\mathrm{r}} \mathrm{MdM}, \\
& \mathrm{E}=\frac{\mathrm{G}}{\mathrm{r}} \mathrm{M}^{2}=6,674 \cdot 10^{-11} \frac{\mathrm{M}^{2}}{\mathrm{r}}
\end{aligned}
$$

The specific exposure radiation pressure of $\mathrm{MBH}$ pressure $p_{e}\left[\mathrm{~N} \mathrm{~m}^{-2}\right]$ on the cover sphere of radius $r<r_{o}$ may be computed by the way:

$p_{e}=\frac{E}{V}=\frac{3 G}{4 \pi} \frac{M^{2}}{r^{3}}=1.6 \cdot 10^{-11} \frac{M^{2}}{r^{3}}, \quad r>r_{0}$

where, $V=3 / 4 \pi r^{3}$ is volume of the cover sphere.

That way the exposure radiation pressure on sphere has very small value and presses very short time. Conventional gas balloon keeps pressure up $10^{7} \mathrm{~N} \mathrm{~m}^{-2}$ (100 atm). However, the heat impact may be high and AB-Generator design may have the reflectivity cover and automatically open windows for radiation.

Your attention is requested toward the next important result following from Eq. 20 and 21. Many astronomers try to find (detect) the MBH by a $\mathrm{MBH}$ exposure radiation. But this radiation is small, may be detected but for a short distance, does not have a specific frequency and has a variably long wavelength. This may be why during more than 30 years nobody has successfully observed MBH events in Earth environment though the theoretical estimation predicts about 100 of MBH events annually. Observers take note!.

Supporting the MBH in suspended (levitated) state: The fuel injector can support the MBH in suspended state (no contact the MBH with any material surface). 
The maximal suspended force equals:

$$
F=q V_{f}, \quad q=\frac{P_{u}}{c^{2}}, \quad F=\frac{P_{u} V_{f}}{c^{2}}
$$

Where:

$\mathrm{q}=$ Fuel consumption, $\mathrm{kg}$

$\mathrm{V}_{\mathrm{f}}=\mathrm{A}$ fuel speed, $\mathrm{m} \mathrm{sec}^{-1}$

The fuel (plasma) speed $0.01 \mathrm{c}$ is conventionally enough for supporting the MBH in suspended state.

AB-generator as electric generator: When the Hawking radiation reaches the cover as radio microwaves they may be straightforwardly converted to electricity because they create a different voltage between different isolated parts of the cover sphere as in an antenna. Maximal voltage which can produces the radiation wave is:

$\mathrm{w}=\frac{\varepsilon \varepsilon_{0} \mathrm{E}^{2}}{2}+\frac{\mu \mu_{0} \mathrm{H}^{2}}{2}, \quad \mathrm{w}=\frac{\mathrm{P}_{\mathrm{r}}}{\mathrm{c}}$

Where:

$\mathrm{w}=$ Density of radiation energy, $\mathrm{J} \mathrm{m}^{-3}$

$\mathrm{E}=$ Electric intensity, $\mathrm{V} \mathrm{m}^{-1}$

$\mathrm{H}=$ Magnetic intensity, $\mathrm{T}$

$\varepsilon_{0}=8.85 \times 10^{-12} \mathrm{~F} \mathrm{~m}^{-1}$ is the coefficient of the electric permeability

$\mu_{o}=4 \pi \times 10^{-7} \mathrm{~N} / \mathrm{A}^{2}$ is the coefficient of the magnetic permeability; $\varepsilon=\mu=1$ for vacuum

Let us take moment when $\mathrm{H}=0$, then:

$\mathrm{E}=\sqrt{\frac{2 \mathrm{~W}}{\varepsilon_{0}}}=\sqrt{\frac{2 \mathrm{P}_{\mathrm{r}}}{\varepsilon_{0} \mathrm{c}}}=2.73 \sqrt{\mathrm{P}_{\mathrm{r}}} \quad \mathrm{U} \approx \mathrm{bDE}, \quad \mathrm{b}=\frac{\mathrm{D}}{\lambda} \leq 1$,

$\mathrm{P}_{\mathrm{e}} \approx \mathrm{bP}_{\mathrm{r}}, \quad \lambda=\lambda_{0} \frac{\mathrm{r}}{\mathrm{r}_{0}}=16 \mathrm{r}, \quad \mathrm{b}=\frac{2 \mathrm{r}}{16 \mathrm{r}}=\frac{1}{8}$

Where:

$\mathrm{E}=$ Electric intensity, $\mathrm{V} \mathrm{m}^{-1}$

$\mathrm{U}=$ Voltage of $\mathrm{AB}$-generator, $\mathrm{V}$

$\mathrm{B}=$ Relative size of antenna

$\mathrm{D}=$ Diameter of the cover sphere if the cover sphere is used as a full antenna, $m$

$\mathrm{P}_{\mathrm{e}}=$ Power of the electric station, $\mathrm{W}$

As you see about $1 / 8$ of total energy produced by AB-Generator we can receive in the form of electricity and $7 / 8$ ths reflects back to $\mathrm{MBH}$; we may tap heat energy which convert to any form of energy by conventional (heat engine) methods. If we reflect the most part of the heat energy back into the $\mathrm{MBH}$, we can have only electricity and do not have heat flux.

If we will use the super strong and super high temperature material $\mathrm{AB}$-material offered $\mathrm{in}^{[3]}$ the conversion coefficient of heat machine may be very high.

Critical mass of $\mathrm{MBH}$ located in matter environment: Many people are afraid the $\mathrm{MBH}$ experiments because $\mathrm{BH}$ can absorb the Earth. Let us find the critical mass of $\mathrm{MBH}$ which can begin uncontrollably to grow into the Earth environment. That will happen when $\mathrm{BH}$ begins to have more mass than mass of Hawking radiation. Below is the equation for the critical mass of initial $\mathrm{BH}$. The educated reader will understand the equations below without detailed explanations:

$$
\begin{aligned}
& \mathrm{dV} \quad=\mathrm{gdt}, \quad \mathrm{g}=\frac{\mathrm{GM}}{\mathrm{r}^{2}}, \quad \mathrm{dt}=\frac{\mathrm{dr}}{\mathrm{V}}, \quad \mathrm{VdV}=\mathrm{gdr}, \\
& \int_{\mathrm{V}}^{\mathrm{c}} \mathrm{VdV}=\int_{\mathrm{r}}^{\mathrm{r}_{\mathrm{o}}} \frac{\mathrm{GM}}{\mathrm{r}^{2}} \mathrm{dr}, \quad \mathrm{r}_{0}=\frac{2 \mathrm{G}}{\mathrm{c}^{2}} \mathrm{M}, \quad \mathrm{V}^{2}=\mathrm{c}^{2} \frac{\mathrm{r}_{0}}{\mathrm{r}}, \\
& \mathrm{V} \quad=\mathrm{c} \sqrt{\frac{\mathrm{r}_{0}}{\mathrm{r}}}, \quad \mathrm{dt}=\frac{\sqrt{\mathrm{r} d r}}{\mathrm{c} \sqrt{\mathrm{r}_{0}}}, \quad \int_{\mathrm{t}}^{0} \mathrm{dt}=\frac{1}{\mathrm{c} \sqrt{\mathrm{r}_{0}}} \int_{\mathrm{r}}^{\mathrm{r}_{0}} \sqrt{\mathrm{r}} \mathrm{dr}, \\
& \mathrm{t} \quad=\frac{2}{3 \mathrm{c} \sqrt{\mathrm{r}_{0}}}\left(\mathrm{r}^{3 / 2}-\mathrm{r}_{0}^{3 / 2}\right) \approx \frac{2 \mathrm{r}^{3 / 2}}{3 \mathrm{cr} \mathrm{r}_{0}^{1 / 2}}, \quad \mathrm{r}=\left(\frac{3 \mathrm{c} \sqrt{\mathrm{r}_{0}}}{2} \mathrm{t}\right)^{3 / 2} \text {, } \\
& r \quad=1.65 \mathrm{G}^{1 / 2} \mathrm{M}^{1 / 3} \mathrm{t}^{2 / 3}, \quad \dot{\mathrm{M}}=\frac{\mathrm{P}}{\mathrm{c}^{2}}=\frac{\hbar \mathrm{c}^{4}}{15360 \pi \mathrm{G}^{2}} \frac{1}{\mathrm{M}^{2}} \\
& =4 \cdot 10^{15} \frac{1}{\mathrm{M}^{2}}, \quad \text { for } \mathrm{t}=1 \mathrm{~s} \text {, }
\end{aligned}
$$

Where:

$\mathrm{V}=$ Speed of environment matter absorbed by $\mathrm{MBH}, \mathrm{m} \mathrm{sec}^{-1}$

$\mathrm{g}=$ Gravity acceleration of $\mathrm{MBH}, \mathrm{m} \mathrm{sec}^{-1}$

$\mathrm{r}=$ Distance environment matter to $\mathrm{MBH}$ center, $\mathrm{m}$

$\mathrm{t} \quad=$ Time, sec

$\dot{\mathrm{M}}=$ Mass loss by MBH, $\mathrm{kg}$

$\dot{\mathrm{M}}_{\mathrm{e}}=$ Mass taken from Earth environment by $\mathrm{MBH}, \mathrm{kg}$

$\gamma=$ Density of Earth environment, $\mathrm{kg} \mathrm{m}^{-3}$

$\mathrm{M}_{\mathrm{c}}=$ Critical mass of $\mathrm{MBH}$ when one begin uncontrollable grows, $\mathrm{kg}$

$\mathrm{t}=$ Time, sec 
Let us to equate the mass $\dot{\mathrm{M}}$ radiated by $\mathrm{MBH}$ to mass $\dot{\mathrm{M}}_{\mathrm{e}}$ absorbed by $\mathrm{MBH}$ from Earth environment, we obtain the critical mass $\mathrm{M}_{\mathrm{c}}$ of $\mathrm{MBH}$ for any environment:

$$
\begin{aligned}
\mathrm{M}_{\mathrm{c}}^{3} & =\frac{\hbar \mathrm{c}^{4}}{92160 \pi^{2} \mathrm{G}^{3}} \frac{1}{\gamma}=3.17 \cdot 10^{24} \frac{1}{\gamma}, \quad \text { or } \\
\gamma & =3.17 \cdot 10^{24} \frac{1}{\mathrm{M}_{\mathrm{c}}^{3}}
\end{aligned}
$$

If $\mathrm{MBH}$ having mass $\mathrm{M}=10^{7} \mathrm{~kg}$ (10 thousands tons) is put in water $\left(\gamma=1000 \mathrm{~kg} \mathrm{~m}^{-3}\right)$, this MBH can begin uncontrollable runaway growth and in short time (Earth $\sim 74 \mathrm{sec}$ ) can consume the Earth into a black hole having diameter $\sim 9 \mathrm{~mm}$. If this $\mathrm{MBH}$ is located in the sea level atmosphere $\left(\gamma=1.29 \mathrm{~kg} \mathrm{~m}^{-3}\right)$, the initial $\mathrm{MBH}$ must has critical mass $\mathrm{M}=10^{8} \mathrm{~kg}$ (100 thousand tons). The critical radius of $\mathrm{MBH}$ is very small. In the first case $\left(M=10^{7} \mathrm{~kg}\right) \mathrm{r}_{\mathrm{o}}=1.48 \times 10^{-20} \mathrm{~m}$, in the second case $\left(\mathrm{M}=10^{8} \mathrm{~kg}\right) \mathrm{r}_{\mathrm{o}}=1.48 \times 10^{-19} \mathrm{~m}$. That is less in $10 \div 100$ thousands times them an atom nuclei. Our $\mathrm{MBH}$ into $\mathrm{AB}-G e n e r a t o r$ is not dangerous for Earth because it is located in vacuum and has mass thousands to millions times less than the critical mass.

However, in a moment of extreme speculation, if far future artificial intelligence (or super-small reasoning) beings will be created from nuclear matter ${ }^{[3]}$ they can convert the Earth into a black hole to attempt to access quick travel to other stars (Solar systems), past and future Universes and even possibly past and future times.

General note: We got our equations in assumption $\lambda / \lambda_{\mathrm{o}}=\mathrm{r} / \mathrm{r}_{\mathrm{o}}$. If $\lambda / \lambda_{\mathrm{o}}=\left(\mathrm{r} / \mathrm{r}_{\mathrm{o}}\right)^{0.5}$ or other relation, the all above equations may be easy modified.

Project of AB-generator: Let us to estimate the possible energy production of an AB-Generator. That is not optimal, that is example of computation and possible parameters. Let us take the $\mathrm{MBH}$ mass $\mathrm{M}=10^{-5} \mathrm{~kg}$ and radius of the cover sphere $\mathrm{r}=5 \mathrm{~m}$. No reflection. Using the Eq. 1-24 we receive:

$$
\begin{aligned}
& \mathrm{P}=3.56 \cdot 10^{32} / \mathrm{M}^{2}=3,56 \cdot 10^{42} \quad \mathrm{~W}, \\
& \mathrm{r}_{0}=1.48 \cdot 10^{-27} \mathrm{M}=1.48 \cdot 10^{-32} \mathrm{~m}, \\
& \xi=\mathrm{r}_{0} / \mathrm{r}=2.96 \cdot 10^{-33}, \\
& \mathrm{P}_{\mathrm{r}}=\xi \mathrm{P}=1.05 \cdot 10^{10}, \quad \mathrm{P}_{\mathrm{u}}=\xi \xi_{\mathrm{r}} \mathrm{P}=\mathrm{P}_{\mathrm{r}}, \quad \mathrm{W}, \quad \xi_{\mathrm{r}}=1 . \\
& \lambda_{0}=3,73 \cdot 10^{-26} \mathrm{M}=3.73 \cdot 10^{-31} \quad \mathrm{~m} . \\
& \lambda=16 \cdot \mathrm{r}=80 \mathrm{~m} . \\
& \mathrm{p}=\frac{\mathrm{P}_{\mathrm{r}}}{4 \pi \mathrm{cr}^{2}}=0.111 \quad \frac{\mathrm{N}}{\mathrm{m}^{2}}, \quad \mathrm{c}=3 \cdot 10^{8} \quad \mathrm{~m} \mathrm{sec}^{-1}, \\
& \dot{\mathrm{M}}=\mathrm{P}_{\mathrm{u}} / \mathrm{c}^{2}=1.17 \cdot 10^{-7} \quad \mathrm{~kg} \mathrm{sec}{ }^{-1}, \\
& \mathrm{p}_{\mathrm{e}}=1.6 \cdot 10^{-11} \frac{\mathrm{M}^{2}}{\mathrm{r}^{3}}=1.28 \cdot 10^{-23} \quad \mathrm{~N} \mathrm{~m}^{-2}
\end{aligned}
$$

Remain the main notations in Eq. 27: $\mathrm{P}_{\mathrm{r}}=\mathrm{P}_{\mathrm{u}}=$ $1.05 \times 10^{10} \mathrm{~W}$ is the useful energy (1/8 of this energy may be taken as electric energy by cover antenna, $7 / 8$ is taken as heat); $\lambda=80 \mathrm{~m}$ is wavelength of radiation at cover sphere (that is not dangerous for people); $\dot{\mathrm{M}}=1.17 \times 10^{-7} \mathrm{~kg} \mathrm{sec}{ }^{-1}$ is fuel consumption; $\mathrm{r}_{\mathrm{o}}=1.48 \times 10^{-32} \mathrm{~m}$ is radius of $\mathrm{MBH} ; \mathrm{p}_{\mathrm{e}}=1.28 \times 10^{-23} \mathrm{~N} \mathrm{~m}^{-2}$ is explosion pressure of MBH.

Look your attention-the explode pressure is very small. That is less in billions of time then radiation pressure on the cover surface $\mathrm{p}=0.111 \mathrm{~N} \mathrm{~m}^{-2}$. That is no wonder because $\mathrm{BH}$ takes back the energy with that spent for acceleration the matter in eating the matter. No dangerous from explosion of $\mathrm{MBH}$.

Heat transfer and internal electric power are:

$$
\begin{aligned}
& \mathrm{q}=\frac{\mathrm{P}_{\mathrm{u}}}{\mathrm{S}}=\frac{\mathrm{P}_{\mathrm{u}}}{4 \pi \mathrm{r}^{2}}=3.34 \cdot 10^{7} \quad \frac{\mathrm{W}}{\mathrm{m}^{2}}, \\
& \text { For } \delta=2 \cdot 10^{-3} \mathrm{~m}, \quad \lambda_{\mathrm{h}}=100, \\
& \Delta \mathrm{T} \approx \mathrm{q} \delta / \lambda_{\mathrm{h}}=668^{\circ} \mathrm{K}, \\
& \mathrm{E}=2.73 \sqrt{\mathrm{P}_{\mathrm{r}}}=2.8 \cdot 10^{5} \quad \mathrm{~V} / \mathrm{m}, \\
& \mathrm{U}=\mathrm{E} \cdot 2 \mathrm{r}=2.8 \cdot 10^{6} \quad \mathrm{~V}, \\
& \mathrm{P}_{\mathrm{e}}=\mathrm{P}_{\mathrm{r}} / 8=1.31 \cdot 10^{9} \quad \mathrm{~W}
\end{aligned}
$$

Where:

$\mathrm{q}=$ Specific heat transfer through the cover sphere

$\mathrm{S}=$ Internal surface of the cover sphere, $\mathrm{m}^{2}$

$\Delta=$ Thickness of the cover sphere wall, $\mathrm{m}$

$\lambda_{\mathrm{h}}=$ Heat transfer coefficient for steel

$\Delta \mathrm{T}=$ Difference temperature between internal and external walls of the cover sphere

$\mathrm{E}=$ Electric intensity from radiation on cover sphere surface, $\mathrm{V} \mathrm{m}^{-1}$

$\mathrm{U}=$ Maximal electric voltage, $\mathrm{V}$

$\mathrm{P}_{\mathrm{e}}=$ Electric power, $\mathrm{W}$

We get the power heat and electric output of a ABgenerator as similar to a very large complex of present day Earth's electric power stations $\left(\mathrm{P}_{\mathrm{r}}=10^{10} \mathrm{~W}\right.$, ten billion of watts). The AB-Generator is cheaper by a hundred times than a conventional electric station, especially since, we may reflect a heat energy back to the $\mathrm{MBH}$ and not built a heat engine with all the problems of conventional power conversion equipment (using only electricity from spherical cover as antenna).

We hope the Large Hadron Collider at CERN can get the initial $\mathrm{MBH}$ needed for $\mathrm{AB}-$ Generator. The other way to obtain one is to find the Planck $\mathrm{MBH}$ (remaining from the time of the Big Bang and former $\mathrm{MBH})$ and grow them to target $\mathrm{MBH}$ size. 


\section{RESULTS}

- Author has offered the method and installation for converting any conventional matter to energy according the Einstein's equation $\mathrm{E}=\mathrm{mc}^{2}$, where $\mathrm{m}$ is mass of matter, $\mathrm{kg} ; \mathrm{c}=3 \times 10^{8}$ is light speed, $\mathrm{m} \mathrm{sec}^{-1}$

- The Micro Black Hole (MBH) is offered for this conversion

- Also is offered the control fuel guns and radiation reflector for explosion prevention of $\mathrm{MBH}$

- Also is offered the control fuel guns and radiation reflector for the MBH control

- Also is offered the control fuel guns and radiation reflector for non-contact suspension (levitation) of the MBH

- For non contact levitation of MBH the author also offers:

- Controlled charging of MBH and of ends of the fuel guns

- Control charging of rotating MBH and control of electric magnets located on the ends of the fuel guns or out of the reflector-heater sphere

- The author researches show the very important fact: A strong gamma radiation produced by Hawking radiation loses energy after passing through the very strong gravitational MBH field. The MBH radiation can reach the reflector-heater as the light or short-wave radio radiation. That is very important for safety of the operating crew of the AB-Generator

- The author researches show: The matter particles produced by the MBH cannot escape from MBH and can not influence the Hawking radiation

- The author researches show another very important fact: The MBH explosion (hundreds and thousands of TNT tons) in radiation form produces a small pressure on the reflector-heater (cover sphere) and does not destroys the $\mathrm{AB}$-generator (in a correct design of $\mathrm{AB}$-generator!). That is very important for safety of the operating crew of the AB-generator

- The author researches show another very important fact: The MBH cannot capture by oneself the surrounding matter and cannot automatically grow to consume the planet

- As the initial MBH can be used the Planck's (quantum) $\mathrm{MBH}$ which may be everywhere. The offered fuel gun may to grow them (or decrease them) to needed size or the initial MBH may be used the MBH produce Large Hadron Collider (LHC) at CERN. Some scientists assume LHC will produce one $\mathrm{MBH}$ every second $(86,400 \mathrm{MBH}$ in day). The cosmic radiation also produces about $100 \mathrm{MBH}$ every year
- The spherical dome of MBH may convert part of the radiation energy to electricity

- A correct design of MBH generator does not produce the radioactive waste of environment

- The attempts of many astronomers find (detect) the $\mathrm{MBH}$ by a MBH exposure radiation will not be successful without knowing the following: The $\mathrm{MBH}$ radiation is small, may be detected only over a short distance, does not have specific frequency and has a variable long wavelength

\section{DISCUSSION}

We got our equations in assumption $\lambda / \lambda_{\mathrm{o}}=\mathrm{r} / \mathrm{r}_{\mathrm{o}}$. If $\lambda / \lambda_{\mathrm{o}}=\left(\mathrm{r} / \mathrm{r}_{\mathrm{o}}\right)^{0.5}$ or other relation, the all above equations may be easy modified.

The Hawking article was published 34 years ago ${ }^{[1]}$. After this time the hundreds of scientific works based in Hawking work appears. No facts are known which creates doubts in the possibility of Hawking radiation but it is not proven either. The Hawking radiation may not exist. The Large Hadron Collider has the main purpose to create the MBHs and detect the Hawking radiation.

\section{CONCLUSION}

The AB-generator could create a revolution in many industries (electricity, car, ship and transportation). That allows designing photon rockets and flight to other star systems. The maximum possible efficiency is obtained and a full solution possible for the energy problem of humanity. These overwhelming prospects urge us to research and develop this achievement of science.

\section{ACKNOWLEDGEMENT}

The researcher wishes to acknowledge Joseph Friedlander (of Shave Shomron, Israel) for correcting the English and offering useful advice and suggestions.

\section{REFERENCES}

1. Hawking, S.W., 1974. Black hole explosions? Nature, 248: 30-31. DOI: 10.1038/248030a0

2. Bolonkin, A.A., 2006. Non-Rocket Space Launch and Flight. Elsevier, ISBN: 0080447317, pp: 468.

3. Bolonkin, A.A., 2009. Femtotechnology: Nuclear AB-matter with fantastic properties. Am. J. Eng. Applied Sci., 2: 501-514.

4. Wikipedia, Some background material in this article is gathered from Wikipedia under the creative commons license. 